\title{
Dependencia a benzodiazepinas en un centro de atención primaria de salud: Magnitud del problema y orientaciones para el manejo integral Benzodiazepine dependence at a primary care health center: Problem extention and orientations for its global handling
}

Mauricio Olivera V. ${ }^{1}$

Addiction to benzodiazepines is a relatively unknown public health problem so as an extremely complex one between Primary Care Health (PCH) teams. Their usefulness for treatment of anxiety and other disorders, so as their abuse potential, are well known. In several reports, the use between PCH patients is to 30-40\%, with 30\% of self-prescription and $4 \%$ of addiction. $30 \%$ of prescriptions come from PCH physicians and they are frequently not justified. In a primary care center from the southeastern area of Santiago, 1081 Mental Health Program outpatients asked for attention over a six-months seeking period; addiction to benzodiazepines was diagnosed in 111 (10,3\%), according to the DSM-IV criteria. Average age was 60 ys with female-to-male ratio of 3,3:1. 66,6\% of them had at least one comorbidity, 20\% of which was a mood disorder, 12,6\% an anxiety disorder, 7\% panic disorder, 9\% sleep disorder, 4,5\% dementia, 16\% anxious-depresive disorder and $11 \%$ a personality disorder. Some directions for optimus managemente are proposed: determine the severity of addiction; gradual retirement of the drug when possible; careful prescription of BZD and developing and maintaining a solid and stable therapeutic relationship.

Key words: Benzodiazepines; addiction to; Primary Care. Rev Chil Neuro-Psiquiat 2009; 47 (2): 132-137

\section{Introducción}

$\mathrm{D}$ esde la aparición del clordiazepóxido en 1959, más de 3.000 benzodiazepinas (BZD) han sido sintetizadas, 50 de las cuales están actualmente en el mercado. Su utilidad para el control de la ansiedad y como coadyuvante en otros trastornos, tanto en psiquiatría como en la práctica médica general, es ampliamente reconocida, tal como su potencial de abuso y dependencia ${ }^{13,19}$.

La teoría más difundida en relación al mecanismo de acción hipnótico y/o sedante de estas drogas se centra en el GABA como el más conocido depresor endógeno del sistema nervioso cen-

Recibido: 6 de enero de 2009

Aceptado: 18 de marzo de 2009

1 Médico-cirujano general, jefe de la Unidad de Psiquiatría del Hospital San Carlos de Ancud (Chile). 
tral. Se cree que el uso crónico de BZD resulta en una regulación negativa del receptor GABA-inhibidor y cambios configuracionales del complejo receptor-agonista, disminuyendo la sensibilidad al agonista. Estos cambios explicarían los fenómenos de tolerancia, dependencia y síndrome de abstinencia asociados al consumo prolongado de $\mathrm{BZD}^{11}$.

\section{Epidemiología}

En Brasil, la tasa semanal de consumo de tranquilizantes en la población general es de $8 \%$, la gran mayoría por prescripción de médicos generales $^{16}$. En Chile, un estudio poblacional en Santiago encontró una prevalencia anual en el uso de psicofármacos de $31,4 \%$ siendo mayor en mujeres que en hombres (38,9\% vs $22,8 \%$ ). Un $42,9 \%$ de la población había consumido alguna vez en su vida BZD y un $4,1 \%$ podía considerarse como dependiente a las BZD, un porcentaje tres veces más alto que el promedio mundial. Además, se encontró que un $28,9 \%$ de los psicofármacos consumidos eran automedicados ${ }^{6}$. En 1988 en Concepción, se reportó que un 58\% de las BZD se expendían sin prescripción médica y que de la automedicación en mujeres, un $85 \%$ correspondía a ansiolíticos ${ }^{18}$.

El uso de psicofármacos en la atención primaria (APS) en todo el mundo está bien documentado. Una proporción importante de las BZD son indicadas por médicos de APS ${ }^{2,4,6,18}$. En un estudio de la OMS (1995), la mitad de los casos de pacientes con trastornos mentales comunes (TMC) estaba tomando psicofármacos, 26\% BZD y $15 \%$ antidepresivos. En un alto porcentaje, el "tratamiento" no guardaba ninguna relación con el diagnóstico específico del paciente; por ejemplo, sólo uno de cada cinco casos de depresión estaba recibiendo antidepresivos, mientras que la mayoría tomaba tranquilizantes ${ }^{3}$.

En otro estudio en consultorios del área norte de Santiago, la prevalencia anual del uso de psicofármacos fue de 29,4\%, 19,4\% correspondiendo a BZD. De éstas, un $38,8 \%$ eran consumidas sin prescripción médica ${ }^{12}$. Otras experiencias en APS han demostrado un consumo de tranquilizantes cercano al $30 \%{ }^{2}$.

Un estudio más reciente en consultorios urbanos y rurales encontró un $47,6 \%$ de consumo de tranquilizantes entre mujeres consultantes y un $25 \%$ eran consumidoras crónicas ${ }^{3}$.

Según datos del CONACE, el consumo de tranquilizantes es siempre mayor en mujeres y aumenta persistentemente con la edad: 1 de cada 4 mujeres usa tranquilizantes entre los 45 y 64 años ${ }^{7,8}$; en población escolar, el consumo de tranquilizantes se triplica entre $8^{\circ}$ básico y $4^{\circ}$ medio 9

En nuestra experiencia, el abuso y dependencia a BZD constituye un problema de primer orden entre los pacientes ingresados al Programa de Salud Mental, especialmente en aquellos que están en tratamiento psicofarmacológico regular. La magnitud de este fenómeno no se ve reflejada en las estadísticas que se entregan periódicamente al nivel central. En otros centros de salud, este diagnóstico ni siquiera es registrado sistemáticamente.

\section{Material y Métodos}

Para el presente estudio descriptivo, la muestra está constituida por la población total adscrita a un consultorio de la zona suroriente de Santiago $(\mathrm{n}=60.644)$. La población adulta ( 20 años y más) es de 41.527 lo que representa el $68,5 \%$ del total. Los datos han sido extraídos del censo semestral del Programa de Salud Mental de junio del 2005 y representan la actividad realizada entre el $1^{\circ}$ de enero y el 30 de junio del mismo año.

Durante ese período, 1081 pacientes fueron atendidos en el Programa de Salud Mental (1,8\% de la población total).

Utilizando los criterios diagnósticos del DSMIV, que considera básicamente la presencia de fenómenos de tolerancia y abstinencia ${ }^{1}, 111$ pacientes fueron diagnosticados como dependientes a BZD (10,3\% de la submuestra, $0,18 \%$ de la población total y $0,26 \%$ de población adulta). A su 
vez, esta cifra corresponde a un tercio de los pacientes que retiran BZD mensualmente y éstos, aproximadamente el $50 \%$ de aquellos tratados con psicofármacos.

Un análisis de las características demográficas de este grupo demostró que la edad promedio era de 60,5 años (rango: 26-82). 26 pacientes eran hombres $(23,4 \%)$ y el $76,6 \%$ restante, mujeres, con una razón de 3,3:1 mayor que el 2:1 reportado en series internacionales ${ }^{16}$.

El estudio de la comorbilidad en estos pacientes demuestra que 74 de ellos $(66,6 \%)$ tenían al menos un diagnóstico asociado en la esfera psiquiátrica. Cinco $(4,5 \%)$ eran portadores de una demencia, $10(9,0 \%)$ un trastorno del sueño, 3 $(2,7 \%)$ presentaban además una dependencia al alcohol, $8(7,2 \%)$ un trastorno de pánico y 14 $(12,6 \%)$ un trastorno de ansiedad generalizada; $23(20,7 \%)$ tenían un trastorno del ánimo, de los cuales $14(12,6 \%)$ correspondían a una depresión crónica, uno $(0,9 \%)$ a un episodio depresivo severo y uno a un trastorno afectivo bipolar. 18 pacientes $(16,2 \%)$ reunían criterios para un trastorno mixto ansioso-depresivo y 13 (11,7\%) para un trastorno de la personalidad.

\section{Discusión}

Resulta preocupante el hecho de que en un grupo poblacional comparable al grueso de la población beneficiaria de la APS en todo el país, un $10 \%$ de los pacientes atendidos por problemas de salud mental sean portadores de una patología que no está incluida en las estadísticas solicitadas por el nivel central. Tal como se mencionó, es presumible que este diagnóstico no está siendo pesquisado, registrado ni mucho menos tratado en la mayoría de los centros de APS, lo que determina que el problema esté siendo ignorado casi por completo por las autoridades de salud.

La dependencia a las BZD no es un fenómeno inocuo. Existen razones para sospechar que el consumo crónico de altas dosis de estos fármacos se asocia con un mayor riesgo de demencia en

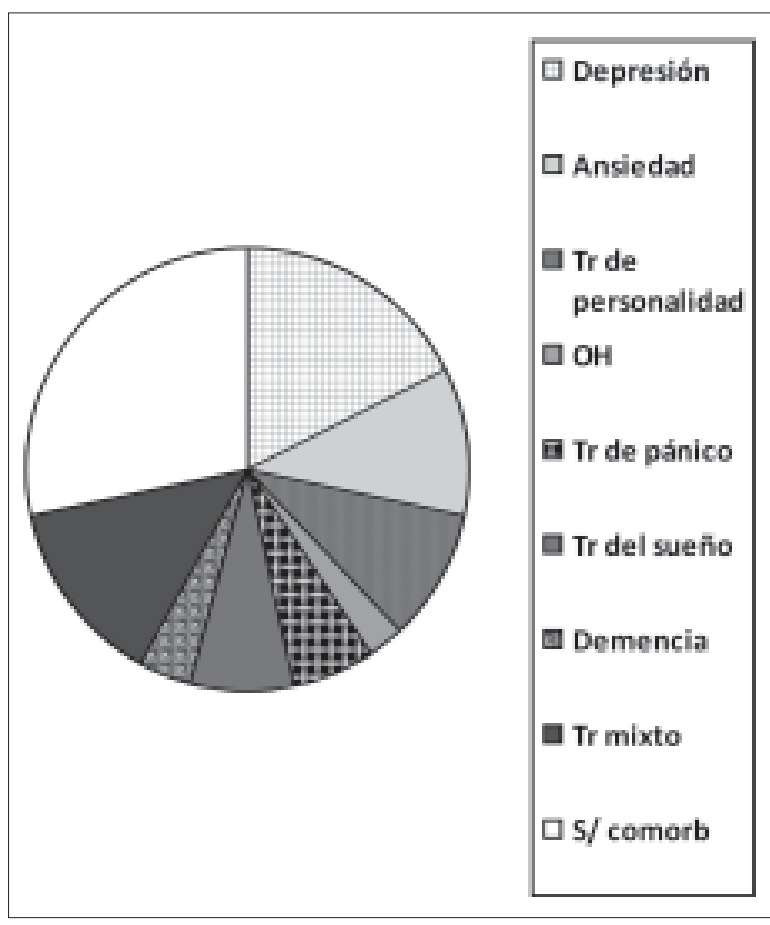

Figura 1. Trastornos comórbidos en pacientes con dependencia a BZD $(\mathrm{n}=111)$.

pacientes de la tercera edad, con las repercusiones obvias para la salud mental, la calidad de vida y el grado de independencia de este grupo etáreo.

Un porcentaje mayoritario de los pacientes dependientes a BZD en la muestra estudiada inician el consumo por prescripción médica, lo que confiere a la inadecuada indicación y/o deficiente control sobre dichos medicamentos un carácter iatrogénico ${ }^{2-4,6,12,15}$. El bajísimo costo de la mayoría de las BZD en el comercio regular favorece la continuidad indefinida del consumo.

Puede apreciarse, en general, que los trastornos que con mayor frecuencia se asociaron al fenómeno de dependencia a BZD en esta muestra fueron de tipo depresivo y ansioso y los trastornos de personalidad. Con respecto a éste último diagnóstico, cabe destacar que un trabajo descriptivo anterior sobre la misma muestra y en el mismo período de tiempo reveló que entre los pacientes con diagnóstico de trastorno de la personalidad, hasta un $20 \%$ presentaban una dependencia a las $\mathrm{BZD}^{17}$. 
También es notorio el hecho de que en un tercio de los pacientes no se identificó ningún otro trastorno mental que justifique el uso prolongado de psicofármacos.

Un adecuado enfrentamiento del fenómeno descrito debe tener en cuenta las siguientes consideraciones:

- Es importante determinar el grado de dependencia de cada paciente, delimitando subgrupos de mayor y menor severidad; como criterios de severidad pueden identificarse: dosis elevadas (tres o más comprimidos diarios), mayor duración del consumo (hasta treinta años en algunos casos), BZD de mayor potencia (alprazolam) o de vida media prolongada (clordiazepóxido, diazepam) y coexistencia de trastornos mentales (depresión crónica, trastornos de ansiedad severos y trastornos de la personalidad). En general, los pacientes de edad avanzada que consumen altas dosis de BZD durante décadas tienen mal pronóstico al plantearse un tratamiento de desintoxicación gradual, en especial si se sospecha u objetiva algún grado de deterioro cognitivo. En estos pacientes, una dosis de mantención estable lo más baja posible pareciera ser el objetivo principal del seguimiento. No debe desconocerse que en el mercado negro, estas mismas drogas son comercializadas a un valor diez veces superior al normal. En todos los demás, debiera ofrecerse la posibilidad de un tratamiento adecuado.

- La desintoxicación debe hacerse en forma gradual, cautelosa y controlada pero decidida, reemplazando la BZD por algún otro psicofármaco para atenuar los síntomas de abstinencia. La recomendación internacional es la dis- minución de un 10-15\% de la dosis diaria o el equivalente a $2 \mathrm{mg}$ de diazepam cada dos semanas, lo que puede tomar hasta seis meses en algunos pacientes. Entre los medicamentos recomendados, los antidepresivos de uso más difundido (fluoxetina y tricíclicos) constituyen el arma de primera línea; también han demostrado utilidad los antihistamínicos, los antipsicóticos, principalmente las fenotiazinas, los sedantes e hipnóticos no benzodiazepínicos, el fenobarbital y los ansiolíticos naturales ${ }^{19}$.

- El predictor más importante de éxito en un proceso de desintoxicación de BZD, así como para cualquier sustancia de abuso, es el establecimiento de una sólida alianza terapéutica basada en la educación sobre la dependencia y sus potenciales consecuencias, la continuidad del tratante y su coherencia en el tiempo, la acogida y el acompañamiento continuo durante todo el proceso, en especial durante la aparición de síntomas de abstinencia ${ }^{5,10,14}$.

- Por último, es indiscutible que la estrategia más valiosa para el enfrentamiento del problema es la prescripción rigurosamente controlada de estos fármacos. La indicación de una BZD por el médico no especialista no debiera superar en ningún caso los 6 meses y en lo posible las 3 semanas. Además, a todo paciente con esta indicación debe explicársele claramente el riesgo de abuso y dependencia, así como sus consecuencias a largo plazo. Debe preferirse siempre un medicamento desprovisto de dichos riesgos para el tratamiento del insomnio o ansiedad como primera línea, así como los antidepresivos para el manejo inicial de los trastornos del ánimo ${ }^{4,15}$. 


\begin{abstract}
Resumen
La dependencia a las benzodiazepinas constituye un problema de salud pública relativamente desconocido y de abordaje complejo para los equipos de Atención Primaria (APS). Son ampliamente conocidos su utilidad en el manejo de distintos trastornos como su potencial de abuso. En diversos estudios, el consumo entre pacientes consultantes en APS alcanza entre 30$40 \%$, con un 30\% de automedicación y 4\% de dependencia en nuestro país. En un 30\% la indicación proviene de un médico de APS y ésta con frecuencia no es pertinente. En un centro de salud de la zona suroriente de Santiago, 1081 pacientes fueron atendidos en el Programa de Salud Mental en un periodo de seis meses; 111 de ellos (10,3\%) fueron diagnosticados como portadores de dependencia a las benzodiazepinas según los criterios del DSM-IV. La edad promedio fue de 60 años, con una proporción por sexos de 3,3 mujeres por 1 hombre. Un 66,6\% de este grupo tenía al menos una patología mental asociada (20\% trastorno del ánimo, 12,6\% trastorno de ansiedad generalizada, 7\% trastorno de pánico, 9\% trastorno del sueño, 4,5\% demencia, $16 \%$ trastorno mixto ansioso-depresivo y $11 \%$ trastorno de la personalidad). Se ofrecen algunas recomendaciones para el adecuado enfrentamiento del problema: determinar la severidad de la dependencia; realizar una desintoxicación gradual en los casos de mejor pronóstico; indicar las BZD con precaución; desarrollar y mantener un vínculo terapéutico sólido y estable.
\end{abstract}

Palabras clave: Benzodiazepinas; dependencia; Atención Primaria.

\section{Referencias}

1. APA: Diagnostic and Statistical Manual of Mental Disorders. 4th ed. Washington, DC: American Psychiatric Association; 1994.

2. Anderson K, Araya R. Benzodiazepines use in Primary HealthCare in Chile. Social Science and Medicine. Submitted.

3. Araya R. Trastornos mentales en la práctica médica general. Biblioteca SAVAL.

4. Beardsley R, Gardocki G, Larson D, Hidalgo J. Prescribing of psychotropic medications by Primary Care physicians and psychiatrists. Arch Gen Psychiatry 1988; 45 (2): 1117-1119.

5. Bernstein J: Handbook of Drug Treatment in Psychiatry. 3rd ed. 1995. Freidman L, Flemming N F: Source book of Substance Abuse and Addiction. 1996.

6. Busto MA. Benzodiazepinas: La tranquila adicción de Santiago. Santiago: CORSAPS, 1991.

7. Chile, Ministerio del Interior, Consejo Nacional para el Control de Estupefacientes (2001). Cuarto Estudio Nacional de Drogas en Población General de Chile, 2000. Santiago de Chile.

8. Chile, Ministerio del Interior, Consejo Nacional para el Control de Estupefacientes (2003). Quinto Estudio Nacional de Drogas en Población General de Chile, 2002. Santiago de Chile.

9. Chile, Ministerio del Interior, Consejo Nacional para el Control de Estupefacientes (2004). Quinto Estudio Nacional de Drogas en Población Escolar de Chile, de $8^{\circ}$ básico a $4^{\circ}$ medio, 2003. Santiago de Chile

10. Galanter M, Kleber HD, eds: Textbook of Substance Abuse Treatment. 3rd ed. American Psychiatric Publishing; 2004.

11. Gilman, Ruddon, Limgird: Goodman \& Gilman: Pharmacological Basis of Therapeutics. 9th ed. 1996.

12. Gonzalez M I, Rojas M G, Díaz B. Uso de psicofármacos por consultantes a nivel primario (comunicación personal). 1993. 
13. Kaplan, Sadock: Comprehensive Textbook of Psychiatry VI. 6th ed. 1995.

14. Karch Steven B, ed: Drug Abuse Handbook. CRC Press: 1997.

15. King M. Is there still a role for benzodiazepines in general practice? British Journal of General Practice 1992; 42: 202-205.

16. Mari J J, Almeida-Filho N, Coutinho E, Baxter Andreoli S, Torres de Miranda C, Streiner D. The epidemiology of psychotropic use in the city of Sao Paulo. Psychological Medicine 1993;
23: 467-479.

17. Olivera M, Galleguillos I, Espinoza M: Protocolo de diagnóstico y tratamiento del trastorno de personalidad limítrofe en Atención Primaria. VIII Congreso de Atención Primaria 2006 Sept.

18. Ruiz I, Offermans J, Lanctot K, Busto U. Comparative study of Benzodiazepine use in Canada and Chile. J Clin Pharmacol 1993; 33: 124-129.

19. Suzuki J: Sedative, Hypnotic, Anxiolytic Use Disorders. eMedicine library. Updated Jun 8 2006.
Correspondencia:

Mauricio Olivera V.

E-mail: hancudsalumental@hotmail.com 